\title{
Answer to the quiz
}

Answer to the quiz on page 37: Electrocardiogram

Correct answers: $3,5,7,8$

This ECG presents case of the atrial bigeminy with different ways of atrioventricular (AV) and HisPurkinje system conduction. In some episodes, atrial

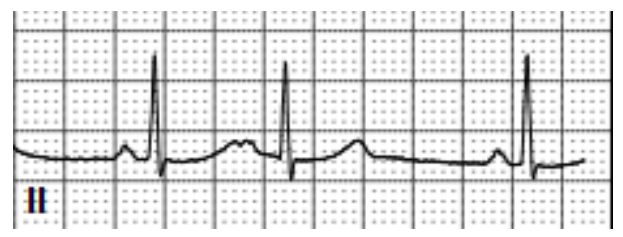

extrasystole is obvious and AV conduction is normal or slightly prolonged (Fig.1):

Figure 1. Atrial bigeminy with varying atrioventricular and His-Purkinje conduction

However, in majority of cases in this ECG strip atrial extrasystoles are concealed. The morphology of the normal $T$ waves (white arrow) is considerably different from morphology of the $T$ waves combined with "hidden" P waves of atrial extrasystoles (gray arrows). The duration of QRS complexes appearing after atrial premature beats in most cases is extended (Fig. 2). There is aberrant conduction via the distal conduction system (His-Purkinje) that is variable and resulting in different morphologies of the QRS complex.

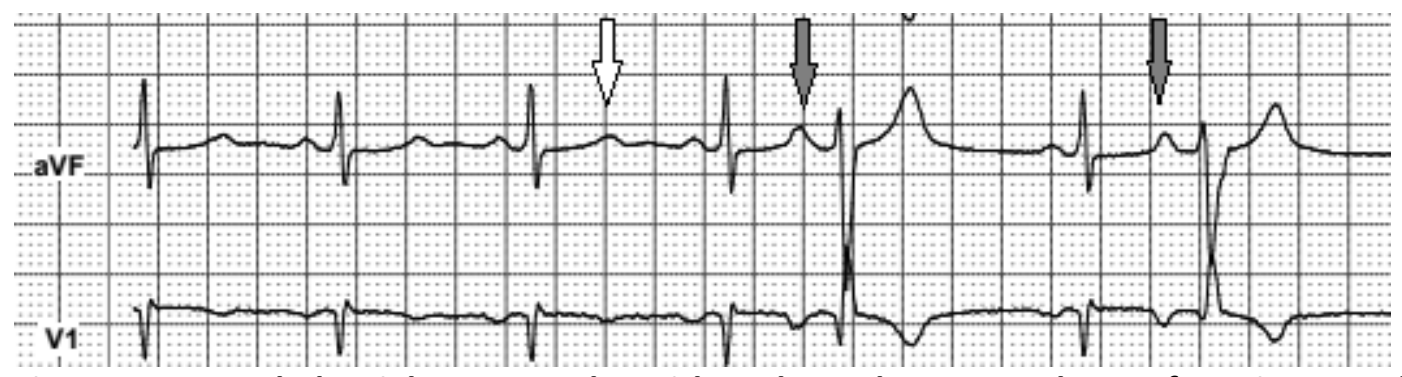

Figure 2. Concealed atrial extrasystoles with prolonged QRS complexes of varying morphology

An episode of prolonged $P Q$ interval can be observed on ECG 2. The duration of the PQ interval (from the beginning of the "hidden" $P$ wave till beginning of the QRS complex) exceeds 200 ms - black marker on
Figure 3. The prolongation of $P Q$ interval can be explained, as conduction delay in both bundle branches of the His-Purkinje system, as seen in trifascicular block.

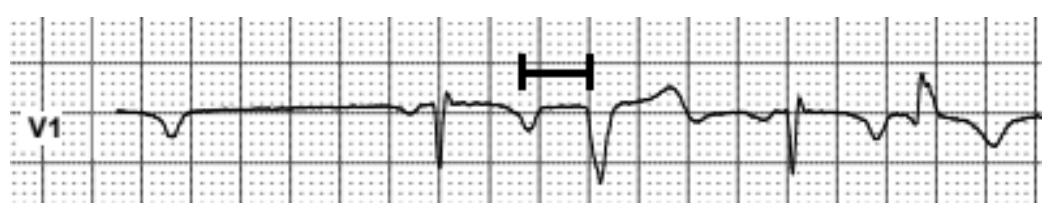

Figure 3. Prolonged PQ interval of an atrial extrasystole beat

Address for correspondence: Mykhaylo Sorokivskyy, Danylo Halytsky Lviv National Medical University, Ukraine Phone: +380677837830 Email: msorokivskyy1@gmail.com

Received: 29.11.2020 Revised: 19.01.2021 Accepted: 20.01.2021

Copyright @2021 Heart, Vessels and Transplantation

Doi: 10.24969/hvt.2021.240 
Finally, the last part of ECG 2 shows slow sinus rhythm. At first sight, considerable sinus bradycardia can be recognized. But after careful comparison of normal $\mathrm{T}$ wave on ECG 1 and altered $\mathrm{T}$ wave morphology on the final part of ECG 2, some important difference can be noticed (Fig. 4).

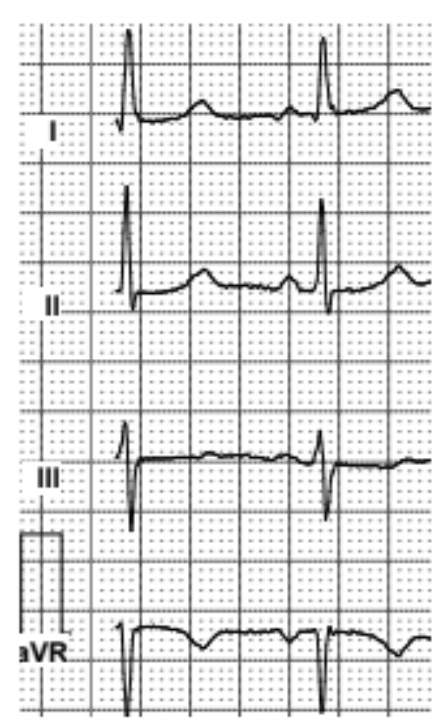

ECG 1

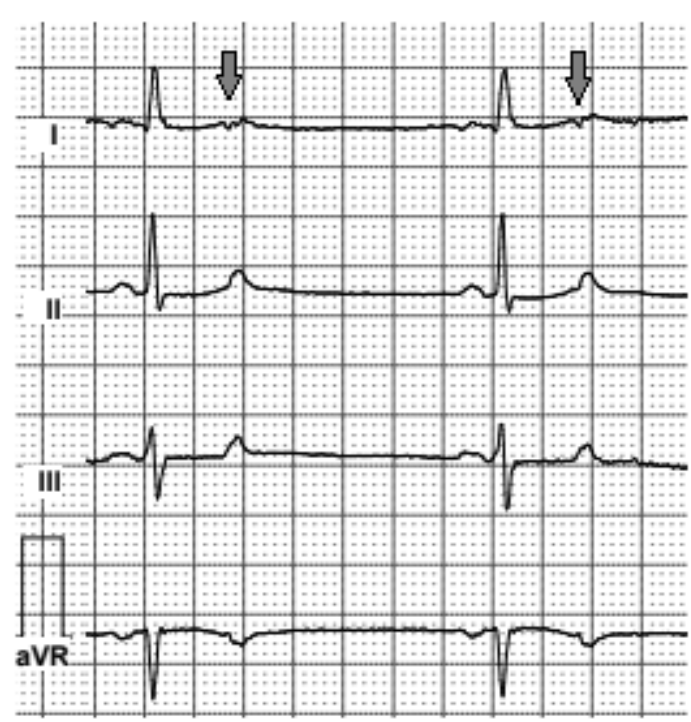

ECG 2

\section{Figure 3. Blocked atrial extrasystoles}

Blocked atrial extrasystoles imitating sinus bradycardia and slowing of heart rate to 42 beats $/ \mathrm{min}$ can be observed on ECG 2 (gray arrows). Blocked atrial bigeminy can cause significant deterioration of the patient's condition causing episodes of lightheadedness and lethargy. Inaccurate ECG analysis in this case can provide to erroneous sinus bradycardia diagnosis. In the worst case, this can lead to inappropriate pacemaker implantation.

On ECG 2, signs of distal conduction delay can be observed. However, Holter recording at higher rates shows normal AV and His-Purkinje conduction, so antiarrhythmic medication could be initiated safely. In case of insufficient effect of antiarrhythmic treatment electrophysiological study and ablation of the atrial ectopic extrasystolia can be considered.

Mykhaylo Sorokivskyy, Rostyslav Kovalchuk, Volodymyr Kulish Danylo Halytsky Lviv National Medical University, Lviv, Ukraine
Conflict of interest: None to declare

Peer-review: Internal and external Authorship: M.S., R.K., V.K. equally contributed to preparation of manuscript and fulfilled authorship criteria Acknowledgement and funding: None to declare 